\title{
Um Modelo Epidemiológico Baseado em Autômatos Celulares Probabilistas Assíncronos
}

\section{Luciano Lucindo Chaves}

Programa de Pós-graduação em Engenharia Elétrica e Computação da Universidade Presbiteriana Mackenzie, São Paulo, SP

\section{Pedro Paulo Balbi de Oliveira ${ }^{2}$}

Faculdade de Computação e Informática da Universidade Presbiteriana Mackenzie, São Paulo, SP

\section{Luiz Henrique Alves Monteiro ${ }^{3}$}

Escola de Engenharia da Universidade Presbiteriana Mackenzie e Escola Politécnica da Universidade de São Paulo, São Paulo, SP

\begin{abstract}
Resumo. Aqui, propõe-se um modelo epidemiológico baseado em autômato celular probabilista assíncrono, no qual os estados de uma fração $\mu$ das células são atualizados somente a cada $\beta>1$ passos de tempo. Mostra-se que os valores de $\mu$ e $\beta$ influenciam o transiente, mas não afetam o regime permanente da dinâmica desse sistema.
\end{abstract}

Palavras-chave. Autômato celular, assincronia, dinâmica populacional, epidemiologia, sistemas dinâmicos.

\section{Introdução}

Epidemiologia teórica consiste no uso de modelos matemáticos no estudo da propagação de patógenos, visando prever a evolução temporal do número de indivíduos infectados, o que é crucial para a elaboração de medidas de controle [1]. No início do século XX, Kermack e McKendrik [8] propuseram um modelo epidemiológico conhecido pela sigla SIR. Descrito por equações diferenciais ordinárias, esse famoso modelo divide a população em três grupos de indivíduos: os suscetíveis, que são os que podem contrair a doença via contatos sociais; os infectados, que podem contaminar os suscetíveis; e os removidos, que são aqueles que tiveram a doença e ou se curaram e ficaram imunes, ou morreram. A sigla SIR decorre das iniciais desses três grupos.

\footnotetext{
$171456457 @$ mackenzista.com.br

2 pedrob@mackenzie.br

3 luizm@mackenzie.br; luizm@usp.br
} 
Teoricamente, pode-se estudar a disseminação de um agente patogênico por meio de equações diferenciais (EDs) ordinárias ou a derivadas parciais [1,10,11]. EDs possibilitam descrever e investigar analiticamente o problema estudado, porém a resolução numérica de tais equações pode apresentar problemas de convergência [10,11].

Autômatos celulares (ACs) têm sido amplamente usados em modelagem epidemiológica, pois permitem representar a geografia da região estudada e as relações sociais

entre os indivíduos de forma natural $[2,3,5,11,13]$. É simples calcular computacionalmente a evolução temporal de um autômato celular; em contrapartida, costuma ser difícil estudá-los analiticamente. Como se percebe, EDs e ACs têm suas vantagens e desvantagens. Talvez, uma abordagem mista seja a mais conveniente $[5,11,13]$.

Em geral, todas as células que compõem o reticulado de um AC têm seus estados atualizados sincronamente; ou seja, a cada e a todo passo de tempo. Porém, alguns trabalhos sugerem que, dependendo do contexto, uma atualização assíncrona pode ser mais realista $[4,6,9]$. Nesse caso, há células que têm seus estados atualizados em instantes diferentes. Nós não conhecemos trabalhos avaliando a influência de assincronia em sistemas epidemiológicos modelados por AC. Aqui, investiga-se essa influência, num modelo do tipo SIS, de modo que a cura não confere imunidade $[1,10]$. Assim, o doente que se curou volta a ser suscetível. Gonorreia é um exemplo típico. No caso dessa doença sexualmente transmissível, assincronia pode refletir a existência de indivíduos que têm relações sexuais com frequência maior do que outros.

Organiza-se este trabalho da seguinte forma. Na Seção 2, apresenta-se o modelo SIS em termos de ACs e EDs. Na Seção 3, descrevem-se os resultados obtidos e comparamse as duas abordagens. Por fim, na Seção 4, expõem-se as conclusões.

\section{O modelo epidemiológico}

Neste trabalho, usa-se um AC probabilista como um modelo epidemiológico do tipo SIS $[1,10,11]$. O reticulado do AC representa a população hospedeira, de modo que cada célula corresponde a um indivíduo que, a cada instante $t$, ou está no estado $I$ (infectado) ou no estado $S$ (suscetível). Há duas regras governando as transições de estados:

1. Regra de contágio: a cada instante $t$, uma célula $S$ pode se tornar $I$ de acordo com a probabilidade $p=1-\exp (-k v)$, sendo $k$ uma constante relacionada à infectividade do patógeno e $v$ a quantidade de células vizinhas infectadas. Quanto maior o valor de $k$ e/ou $v$, maior o valor de $p$. Se a célula $S$ em questão não possui vizinhos infectados, então $p=0$; se $v \rightarrow \infty$ e/ou $k \rightarrow \infty$, então $p \rightarrow 1$.

2. Regra de cura: a cada instante $t$, uma célula $I$ pode se tornar $S$ de acordo com a probabilidade $q$. A transição $I \rightarrow S$ pode também representar a morte de um doente que foi imediatamente compensada pelo nascimento de um suscetível. Segundo essas regras, o tamanho da população permanece constante, o que é conveniente para representar doenças pouco letais e/ou populações em que mortes são compensadas por nascimentos. Um modelo SIR similar foi proposto por Schimit e Monteiro [13].

Aqui, usa-se um reticulado bidimensional de dimensões $n \times n$, com $N=n^{2}$ células, e vizinhança de Moore de raio um (assim, cada célula está conectada às oito células ao seu redor [14]). As condições de contorno são periódicas, de modo que as bordas laterais se conectam assim como se conectam a borda superior e a inferior. Desse modo, tem-se uma superfície toroidal, na qual todas as células possuem o mesmo número de vizinhos. 
A fim de avaliar os efeitos da assincronia na aplicação das regras de transições de estados, estabelece-se que a fração $\mu$ de células sofre atualização de estados somente a cada $t+\beta$ passos de tempo, enquanto que as demais células (a fração $1-\mu$ ) têm seus estados atualizados a cada instante $t$. Essa fração $\mu$ corresponde a uma partição do reticulado que delimita os indivíduos que são atualizados assincronamente. Nos passos de tempo em que não são atualizados, os indivíduos dessa subárea assíncrona permanecem no estado em que se encontram. Ainda assim, eles podem exercer influência sobre os vizinhos, caso estes venham a ter seus estados atualizados.

No caso em que $\mu=0$ e $\beta=0$ (ou seja, sem assincronia), uma aproximação de campo médio para esse modelo pode ser descrita pelas seguintes EDs [10]:

$$
\begin{aligned}
d S(t) / d t & =-a S(t) I(t)+b I(t) \\
d I(t) / d t & =a S(t) I(t)-b I(t)
\end{aligned}
$$

sendo $a$ a constante de taxa de contágio e $b$ a constante de taxa de cura. As variáveis $S(t)$ e $I(t)$ denotam, respectivamente, a quantidade de indivíduos suscetíveis e de infectados no instante $t$. Como $d S(t) / d t+d I(t) / d t=0$, então $S(t)+I(t)=N$, sendo $N$ o número total de indivíduos da população hospedeira. Logo, $S(t)=N-I(t)$. Assim, tem-se que:

$$
d I / d t=F(I)=I[a N-a I-b]
$$

Essa equação possui dois pontos de equilíbrio, obtidos impondo-se que $F\left(I^{*}\right)=0$. Tais pontos são $I^{*}=0$ (chamado de equilíbrio livre de doença) e $I^{*}=(a N-b) / a$ (chamado de equilíbrio endêmico). Para esse sistema não linear de primeira ordem, a derivada de $F(I)$ calculada em $I^{*}$ (ou seja, o autovalor $\lambda$ da matriz jacobiana do sistema linearizado em torno de $\left.I^{*}\right)$ dá a estabilidade de $I^{*}[7,10]$. De fato, $I^{*}$ é assintoticamente estável se $\lambda=$ $d F(I) /\left.d I\right|_{I=I^{*}}<0$ e é instável se $\lambda=d F(I) /\left.d I\right|_{I=I^{*}}>0$. Como $\lambda=a N-2 a I-b$, então para $I^{*}=0$, tem-se que $\lambda=a N-b$; e para $I^{*}=(a N-b) / a$, tem-se que $\lambda=-a N+b$. Portanto, se $a N<b$, a solução estacionária livre de doença é assintoticamente estável e a solução estacionária endêmica é instável; se $a N>b$, essas soluções trocam de estabilidade, o que caracteriza a ocorrência de uma bifurcação transcrítica em $a N=b[7,10]$.

A seguir, apresentam-se resultados de simulações numéricas com o AC e comparamse esses resultados com aqueles derivados do modelo escrito em termos de EDs.

\section{Simulações numéricas e comparações}

Nas simulações numéricas com o AC, consideraram-se os seguintes valores: $k=1, q=0,5$ (assim, um infectado tem 50\% de chance de se tornar suscetível a cada instante em que a regra de transição $I \rightarrow S$ é aplicada), $n=200$ (desse modo, a população é formada por $N=40000$ indivíduos), $I(0)=0,005$ e $S(0)=0,995$ (ou seja, inicialmente a população é constituída de $99,5 \%$ de suscetíveis e de $0,5 \%$ de infectados). O objetivo é investigar os efeitos dos parâmetros $\beta$ e $\mu$ sobre a dinâmica do sistema.

Inicialmente, investigou-se como os valores desses parâmetros afetam o comportamento transiente (isto é, antes de o número de infectados se estabilizar). Para isso, calculou-se o tempo de acomodação $T_{a}$, como sendo o instante em que $I(t)$ atinge pela primeira vez um valor compreendido na faixa que vai de $95 \%$ a $105 \%$ de seu valor médio em regime permanente $[10,12]$. Desse instante em diante, $I(t)$ permanece dentro dessa faixa. 
A Figura 1 exibe a variação de $T_{a}$ em função de $\mu$ e em função de $\beta$. À esquerda, nota-se que, para $\beta=5, T_{a}$ cresce com $\mu$; à direita, percebe-se que, para $\mu=40 \%, T_{a}$ cresce com $\beta$. Portanto, quanto maior a região da subáera assíncrona (dada por $\mu$ ) ou quanto maior o intervalo de tempo no qual a células assíncronas têm seus estados atualizados (dado por $\beta$ ), maior o tempo de acomodação; ou seja, mais longo é o transiente. Cada ponto dessas curvas de $T_{a}$ corresponde ao valor médio de 20 simulações.
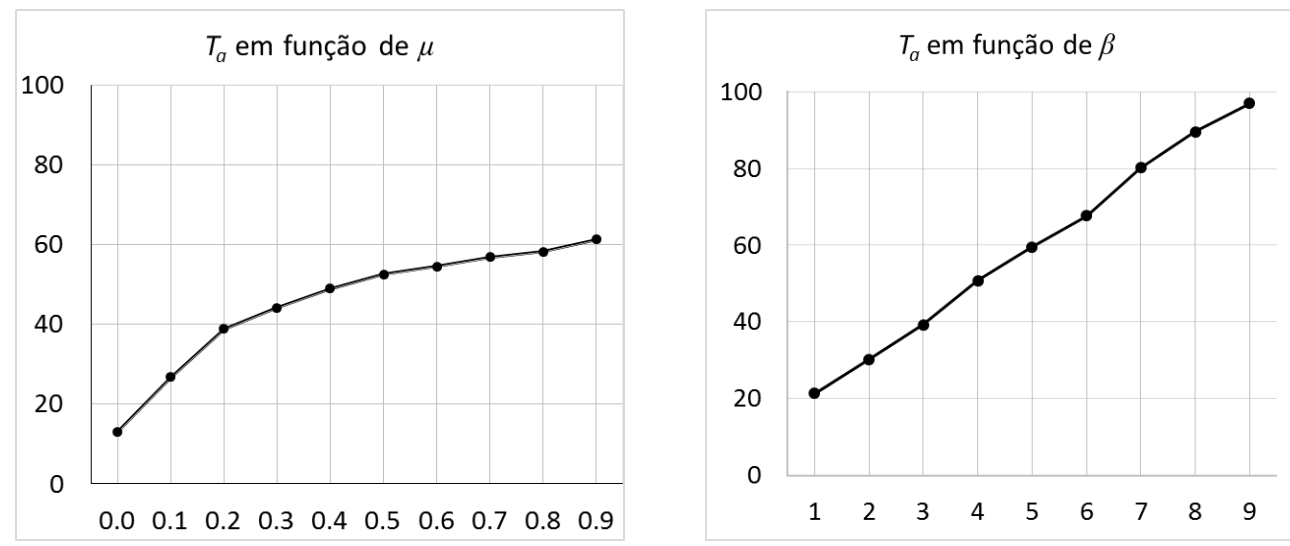

Figura 1: Tempo de acomodação (em passos de tempo) para $k=1, q=1 / 2, n=200$, $I(0)=0,005$ e $S(0)=0,995$. À esquerda, $\beta=5$; à direita, $\mu=0,4$.

A Figura 2 ilustra como varia a quantidade média de infectados, após o AC entrar em regime permanente. Na figura à esquerda, tomou-se $\beta=5$ e variou-se $\mu$; na figura à direita, tomou-se $\mu=40 \%$ e variou-se $\beta$. Note que a porcentagem $\mu$ e o intervalo de tempo $\beta$ referente às células assíncronas não afetam significativamente os valores de infectados no regime permanente. Cada ponto dessas curvas corresponde ao valor médio de 20 simulações.
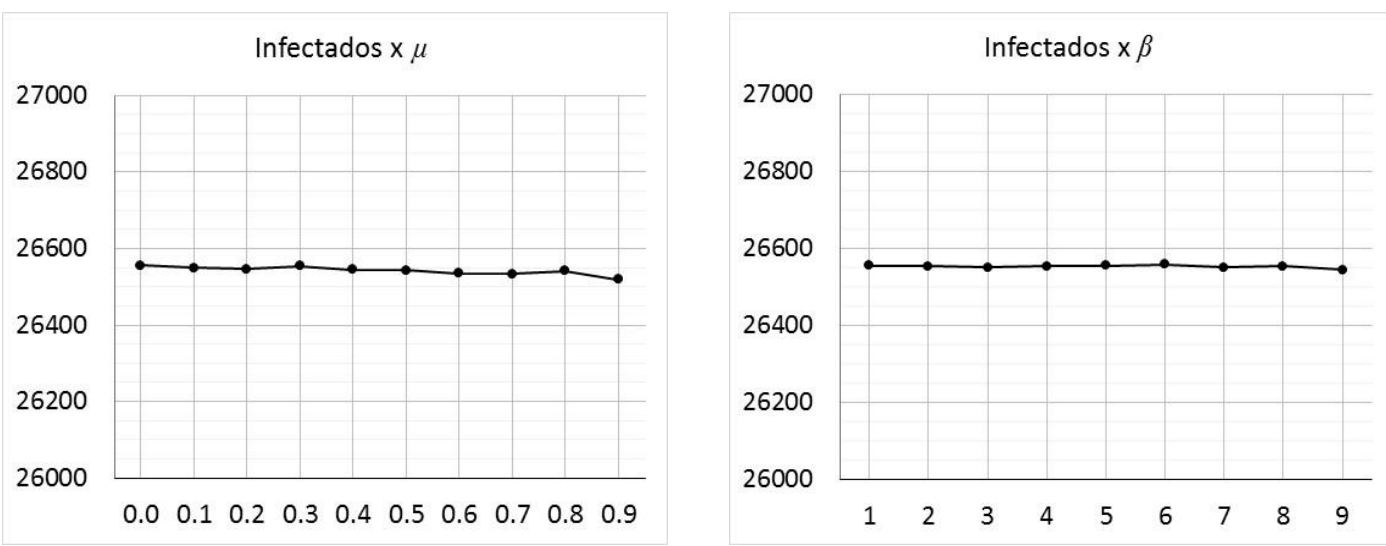

Figura 2: Número médio de infectados para $k=1, q=1 / 2, n=200, I(0)=0,005$ e $S(0)=0,995$. À esquerda, $\beta=5$; ̀̀ direita, $\mu=0,4$. 
É possível estimar os valores dos parâmetros das EDs a fim de que elas reproduzam a dinâmica do AC $[11,13]$. Para o modelo aqui analisado, essa estimativa é feita assim:

$$
\begin{aligned}
& a \approx \Delta(S \rightarrow I) / S^{\prime} I^{\prime} \\
& b \approx \Delta(I \rightarrow S) / I^{\prime} \approx q
\end{aligned}
$$

sendo $\Delta(S \rightarrow I)$ a quantidade média de transições de suscetível para infectado, por passo de tempo, após o sistema entrar em regime permanente, $\Delta(I \rightarrow S)$ a quantidade média de transições de infectado para suscetível, por passo de tempo, após o sistema entrar em regime permanente, e $S^{\prime}$ e $I^{\prime}$ os valores médios de suscetíveis e de infectados, respectivamente, em regime permanente. Aqui, as médias são referentes a 40 passos de tempo.

Considerando os resultados de simulações do AC, foram obtidos os seguintes valores médios em regime permanente: $S^{\prime} \approx 13440, I^{\prime} \approx 26560$ (note que $S^{\prime}+I^{\prime}=40000$ ), $\Delta(S \rightarrow I) \approx 13275$ e $\Delta(I \rightarrow S) \approx 13274$. Calculando os valores de $a$ e $b$, conforme as Equações (4) e (5), obtêm-se $a \approx 0,000037$ e $b \approx 0,50$. De acordo com a Equação (3), o equilíbrio endêmico é dado por $I^{*}=(a N-b) / a=26486$. Note que $a N \approx 1,5>b \approx 0,5$ (que é a condição para tal equilíbrio ser assintoticamente estável). Como $\mu$ e $\beta$ não influenciam o regime permanente, a solução estacionária endêmica calculada a partir da Equação (3) concorda, de fato, com aquelas obtidas via simulações do AC e exibidas na Figura 2.

\section{Conclusões}

Aqui, investigou-se a influência de assincronia na atualização de estados de um modelo epidemiológico do tipo SIS baseado em AC probabilista. Concluiu-se que, para o modelo investigado, a assincronia não afeta o número de infectados em regime permanente; entretanto, quanto maior o percentual $\mu$ de células atualizadas assíncronamente ou quanto maior o intervalo de tempo $\beta$ em que essa atualização é realizada, maior o tempo de acomodação. Portanto, assincronia não afeta o valor de $I^{*}$ a ser atingido, mas afeta como esse valor é atingido. Para eliminar o patógeno da população, a condição que deve ser satisfeita é $a N<b$, já que nesse caso o ponto de equilíbrio livre de doença é assintoticamente estável. Portanto, medidas de controle devem visar alterações nos valores dos parâmetros $a, b$ e/ou $N$, a fim de se ter $a N<b$. Essa condição foi determinada a partir do modelo epidemiológico equivalente escrito em termos de EDs.

A validação desses resultados depende da comparação com dados epidemioló gicos reais. Pretende-se fazer isso em breve.

\section{Agradecimentos}

Luciano L. Chaves é bolsista de doutorado da UPM. Luiz H. A. Monteiro agradece ao CNPq pela bolsa de produtividade. 


\section{Referências}

[1] R. M. Anderson and R. M. May, Infectious Diseases of Humans, Oxford University Press (1991).

[2] E. Ahmed, H. N. Agiza and S. Z. Hassan, On modeling hepatitis B transmission using cellular automata, J. Stat. Phys., vol. 92, 707-712, (1998).

[3] J. R. Doran and S. W. Laffan, Simulating the spatial dynamics of foot and mouth disease outbreaks in feral pigs and livestock in Queensland, Australia, using a susceptible-infected-recovered cellular automata model, Prev. Vet. Med., vol. 70, 133-152 (2005).

[4] N. Fatés, A guided tour of asynchronous cellular automata, J. Cell. Autom., vol. 9, 387-416, (2014).

[5] M. A. Fuentes and M. N. Kuperman, Cellular automata and epidemiological models with spatial dependence, Physica A, vol. 267, 471-486, (1999).

[6] C. Grilo and L. Correia, Effects of asynchronism on evolutionary games, J. Theor. Biol., vol. 269, 109-122, (2011).

[7] J. Guckenheimer and P. Holmes, Nonlinear Oscillations, Dynamical Systems, and Bifurcations of Vector Fields, Springer (2002).

[8] W. O. Kermack and A. G. McKendrick, A contribution to the mathematical theory of epidemics, Proc. Roy. Soc. Lond. A, vol. 115, 700-721, (1927).

[9] L. Manzoni, Asynchronous cellular automata and dynamical properties, Nat. Comput., vol. 11, 269-276, (2012).

[10] L. H. A. Monteiro, Sistemas Dinâmicos, Livraria da Física (2011).

[11] L. H. A. Monteiro, Sistemas Dinâmicos Complexos, Livraria da Física (2014).

[12] K. Ogata, Modern Control Engineering, Prentice-Hall (1997).

[13] P. H. T. Schimit and L. H. A. Monteiro, On the basic reproduction number and the topological properties of the contact network: an epidemiological study in mainly locally connected cellular automata, Ecol. Model., vol. 220, 1034-1042, (2009).

[14] S. Wolfram, Cellular Automata and Complexity: Collected Papers. Westview Press (1994). 